\section{Repeated vs varied distractors in immediate memory*}

\author{
IAN E. GORDON and PAUL FENOULHET \\ University of Exeter, Exeter, England
}

Ss heard groups of eight spoken digits presented for immediate recall. Irrelevant distractor letters were interpolated between digits, using either the same letter interpolated eight times or eight different letters. The latter condition produced significantly more errors in recall. Error scores were also affected by rate of presentation of items and by whether letter-digit sequences started with a letter or a digit. It is suggested that selective immediate recall may bear some resemblance to aspects of visual search.

Morton (1968) and Crowder and Morton (1969) have described a model of memory, the earliest stage of which, in the auditory mode, is an echoic short-term buffer store. Information in the store remains coded in a sensory form. Storage of items occurs prior to their recognition and categorization ("precategorical acoustic storage," or "PAS"), and items are subject to loss by overwriting or decay. The storage time of PAS is of the order of a few seconds, and the store has limited capacity.

In a series of tests of the model, Morton (1968) has described experiments in which six irrelevant distractor items (digits) were interleaved with six relevant items (letters) presented for immediate recall. Performance on letter-digit sequences was significantly poorer than performance on digit-letter sequences. This difference between the two types of lists was interpreted by Morton as an extension of the redundant suffix effect (Dallett, 1964), which can be demonstrated when a single redundant terminal item is added to a list presented for immediate recall, such a suffix typically producing an impairment in the recall of relevant items. Morton's data support his prediction that, as PAS is limited in capacity, its contribution to the recall of the earlier items in a list will be relatively small, and, hence, the differences between relevant-irrelevant and irrelevant-relevant sequences should be progressively smaller towards the earlier serial positions.

In addition to these findings, Morton found that both relevant-irrelevant sequences and irrelevant-relevant sequences produced significantly more errors than did control conditions using six letters only or six letters, each repeated twice.

These experiments raise the general question of how Ss deal with irrelevant distractors during immediate recall. The

*A similar experiment to the one reported here was initially carried out by Henry Shaffer and Jane Hardwick of Exeter University. experiment reported below was designed (1) to extend Morton's technique to longer lists and (2) to see if Ss are sensitive to differences in the composition of the irrelevant distracting material; in particular, if different irrelevant items produce greater impairment in recall than the same irrelevant item repeated several times. In the present experiment, recall items were digits and the irrelevant distractor items were letters.

Materials

Tape recordings were made of 24 lists of spoken letters and digits. The lists varied in three ways:

(1) The variety of letter distractors. Each list was formed by interleaving eight nonrepeating random digits with either eight occurrences of the same letter or eight different letters. The letters used were restricted to the following random selection: B, C, E, G, H, M, R, V.

(2) Relative position of distractors. Half of the lists started with a digit (digit-letter), half with a letter (letter-digit).

(3) Rate of presentation. There were three rates of presentation: one item per $0.5 \mathrm{sec}$, one item per sec, and one item per $1.5 \mathrm{sec}$.

\section{Experimental Design}

All Ss heard all lists. Four lists were heard at Rate 1 , then four at Rate 2 , and so on, with each rate occurring twice in an A B C C B A design. Each group of four lists contained two lists with digits first and two with letters first. In addition, each group of four lists contained two examples of varied letter distractors and two of factors of relative position and variety of distractors were also counterbalanced.

\section{Procedure}

Ss were tested as a group. They were instructed to write down digits on a standard answer sheet when signaled to do repeated single-letter distractors. These so by a click immediately following the final item of each list. Ss were given advance information as to whether the next list started with a digit or letter and at what rate it would be delivered. Three practice trials were given before the start of the main experiment.

At the end of the experiment Ss were asked to attempt to recall which letter of the alphabet had been used as interpolated distractors.

\section{Subjects}

The 47 Ss, 38 men and 9 women, were students at a local college of education.

\section{RESULTS}

Only those responses occurring in the appropriate serial position were scored correct. Using this criterion, 3,278 errors were recorded. The significance of the differences in error distributions was assessed by an overall chi-square test which examined the distribution of errors as a function of rate, single vs varied distractors, and relative position of distractors. The result of this test $\left(\chi^{2}=35.5, \mathrm{df}=6, \mathrm{p}<.01\right)$ indicated that it was appropriate to undertake subsequent analyses of the separate effects of the experimental variables.

\section{(1) Rate of Presentation}

The faster the rate of presentation of letters and digits, the greater the total number of errors recorded. Total errors were distributed as follows: one item per $0.5 \mathrm{sec}$, errors $=1,348$; one item per sec $=$ 1,000 ; one item per $1.5 \mathrm{sec}=930$. These differences are statistically significant $\left(\chi^{2}=68.8, \mathrm{df}=2, \mathrm{p}<.01\right)$.

\section{(2) Relative Position of Distractors}

Morton's (1968) finding is supported in that, as shown in Fig. 1, more errors were recorded on digit-letter lists than on letter-digit lists. This difference is significant $\left(\chi^{2}=15.1, \mathrm{df}=1, \mathrm{p}<.01\right)$. More importantly, a comparison of the curves shown in Fig. I confirms Morton's prediction that the differences between error distributions on these two types of lists will be progressively smaller at earlier serial positions.

\section{(3) The Variety of Distractors}

A total of 1,566 errors occurred using single repeated distractors; 1,712 errors occurred using varied distractors. Chi-square tests show that this overall difference is significant $\left(\chi^{2}=6.6, \mathrm{df}=1\right.$, $p<.02$ ). However, there is a significant tendency for the difference between single and varied distractors to be greater on digit-letter than on letter-digit lists $\left(\chi^{2}=9.2, \mathrm{df}=1, \mathrm{p}<.01\right)$. Figure 2 shows 


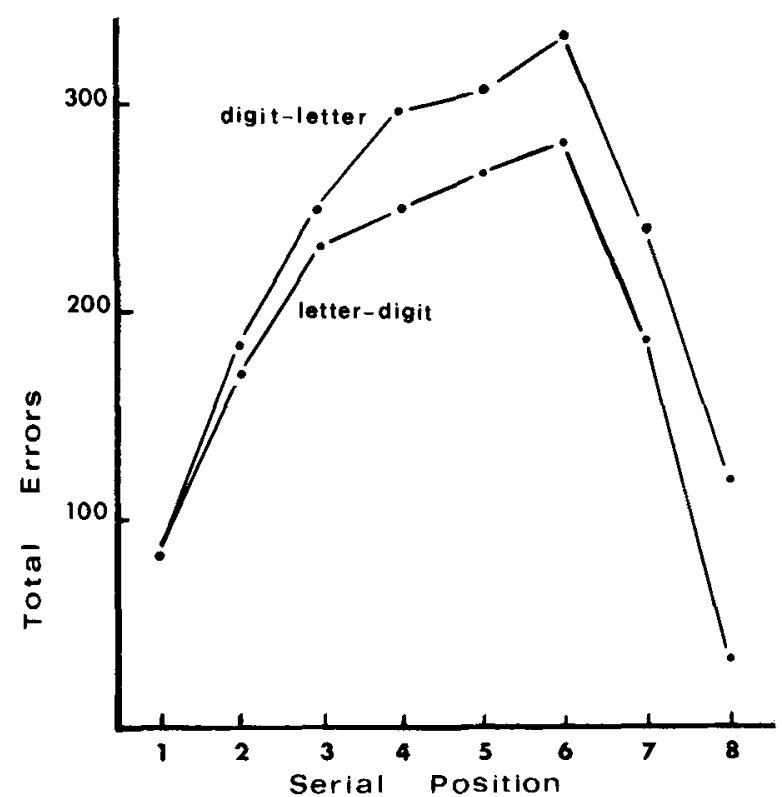

Fig. 1. Serial distribution of errors on letter-digit and digit-letter lists.

the serial distribution of errors using the two kinds of distractor. It can be seen that. with the exception of Position 3, the increase in errors associated with the varied distractors is spread across all serial positions.

\section{(4) Remembering Irrelevant Material}

Only eight letters were used as distractors. Despite the fact that these were heard repeatedly, no $\mathrm{S}$ was able to recall all eight at the end of the experiment. The average number of letters recalled was 4.9 .

\section{DISCUSSION}

Although no single conclusion is forced upon us by the present data, we may speculate as to how Ss deal witlı irrelevant distractor items when these alternate with items presented for immediate recall.

The first possibility is that the irrelevant items are filtered as they are heard, which means, in the present situation, that filtering occurs at the level of the aluditory buffer store (Morton and Crowder's PAS) But if such is the case, why do varied distractors produce greater interference than single repeated distractors particularly as the processing in PAS is believed to be limited to stimulus featuro analysis? And, if irrelevant items are filtered immediately, then performance on lists of relevant and irrelevant items should not differ from performance on contro lists containing the relevant items only. But Morton's (1968) experimental results show that recall using mixed sequences is significantly worse than that of control scquences.

A second possibility is that irrelevant items are stored right up to the point of initiating overt responses, when they are then suppressed. This seems an implausible alternative, particularly as it fails to predict that varied distractors will exert stronger effects. A more important objection, however, is that performance using lists of eight relevant items interleaved with eight irrelevant items is not as poor as might be expected from lists 16 relevant items long, which would be the expected level of difficulty if all 16 items were sustained until the time of actual responding.

The hypothesis offered here as to how distractor items are dealt with originates in a different area of behavior - visual search. Neisser (1964) has shown how, in visual search tasks, various changes in the nature of irrelevant itcms can affect the difficulty of finding relevant (or target) items. An obvious and dependable way of increasing scarch times is to make retevant and irrelevant jtems nore alike. But if has also been shown that the varicty of irrelevant items may influence search times: For example, when lowercase as are hidden in arrays of randomly ordered bs and es, it takes longer to find the as than when ouly bs or only es are used as irrelevant letters. A further increase in search times occurs when the variety of different irrelevant items is raised from two to four (Gordon, 1968).

If the retrieval of items from short-1erm memory at a later stage than PAS has some of the characteristics of a search process, the elfects of varied distractors would be predicted. Further, the demonstration in visual search tasks that $S$ may fail to acquire any lasting information concerning irrelevant material through which they have recently worked (e.g., Neisser \& Beller, 1965; Gordon, 1968) may explain the poor overall level of recall of the distractor items at the end of the present experiment.

We propose that Ss attempting to memorize a number of relevant items from a sequence of relevant and irrelevant items work sequentially through the list, making implicit responses only to relevant items, so that their traces are strengthened relative to the irrelevant items. This process may occur more than once during rehearsal. We can now predict (but not explain) the variety effect. In addition, we can suggest why, in Morton's (1968) experiment, Ss' recall of the list $L, Z, R, X$, $N$. H was worsened by presenting the list in the form $L, L, Z, Z, R, R, X, X, N, N, H, H$,. Morton suggests that such repetition of rclevant itcms interferes with Ss' rehearsal strategies. On the present view any beneficial effect of the repeated presentation of each relevant item will tend to be counteracted by the fact that repetition of items leaves a background of fading traces (varied ones) which have to be searched through during retrieval.

\section{REFERENCES}

CROWDER. R. G., \& MORTON, J. Precategorical acoustic storage (PAS). Pcrception \& Psychophysics, 1969, 5, 365-373.

DALLETT, K. Effects of a redundant prefix on immediate recall. Journal of Experimental Psychology, 1964.67, 296-298.

GORDON, 1. E. Interactions between items in visual search. Journal of Experimental Psychology. 1968, 76, 348-355. 
MORTON, J. Selective interference in immediate

recall. Psychonomic Science, 1968, 12, 75-76. NEISSER, U. Visual search. Scientific American,
June 1964.

NEISSER, U., \& BELLER, H. K. Searching

through word lists. British Journal of
Psychology, 1965, 56, 349-358.

(Accepted for publication August 31, 1970.) 